Baltic Astronomy, vol. 8, 291-300, 1999.

\title{
GAIA SCIENCE OUTPUT: WHITE DWARFS
}

F. Figueras ${ }^{1}$, E. García-Berro ${ }^{2,3}$, J. Torra $^{1,3}$, C. Jordi ${ }^{1}$, X. Luri $^{1}$,

S. Torres ${ }^{4}$ and B. Chen ${ }^{1}$

${ }^{1}$ Departament d'Astronomia i Meteorologia, Universitat de Barcelona, Avda. Diagonal 647, E-08028 Barcelona, Spain

2 Departament de Física Aplicada, Universitat Politècnica de Catalunya, Jordi Girona Salgado s/n Mòdul B-5 Campus Nord, E-08034 Barcelona, Spain

${ }^{3}$ Institut de Estudis Espacials de Catalunya, Edifici Nexus 104, Gran Capità 2-4, E-08034 Barcelona, Spain

4 Departament Telecomunicació i Arquitectura de Computadors, Universitat Politècnica de Catalunya, Avda. Puig Cadafalch 101, E-08303 Mataró, Spain

Received January 25, 1999.

\begin{abstract}
A star count based Galaxy model has been used to estimate the number of disk and halo white dwarfs potentially observable by the ESA astrometric mission Gaia. Using the dependence of the astrometric precision with magnitude and ecliptic latitude, we have evaluated the expected accuracy of the measured distances and tangential velocities of these objects. Our results are a good example what could be the impact of a mission like Gaia on our current understanding of the Galaxy. Scientific attainable goals will include, among others, a reliable determination of the ages of our galactic disk and halo or the reconstruction of the past history of the Star Formation Rate (SFR) of the galactic disk.
\end{abstract}

Key words: astrometry - stars: white dwarfs - orbiting observatories: Gaia 


\section{INTRODUCTION}

White dwarf stars are ideal candidates to probe the history of our Galaxy since these "fossil" stars have very long evolutionary time-scales and, at the same time, their evolution is relatively well understood - at least up to moderately low luminosities. In fact, the white dwarf luminosity function has already become an important tool to determine some properties of the local neighborhood, such as its age (Winget et al. 1987, García-Berro et al. 1988, Hernanz et al. 1994) or the past history of the star formation rate (Noh \& Scalo 1990, Díaz-Pinto et al. 1994, Isern et al. 1995a,b). However, a major drawback of such studies is the small number of white dwarfs accessible to good quality observations.

In this paper we show how an astrometric mission like Gaia could dramatically improve the observational database and, therefore, could have a large impact in our current understanding of the Galaxy. The paper is organized as follows. In Section 2 we describe the computational procedure. In Section 3 we discuss which are the global number of white dwarfs accessible to Gaia, depending on the limiting magnitude and describe our results in detail. Finally in Section 4 we show which science goals could be attainable with the potentially observable database.

\section{SIMULATIONS FROM THE STAR COUNT MODEL}

Our star count Galaxy Model (Chen 1997, Torra et al. 1999) uses the fundamental equation of stellar statistics to predict the number of a given set of stars as a function of their apparent magnitude $V$, and of their $B-V$ and $V-I$ color indices. Structural and kinematical parameters for each population are used to predict the kinematical observables. We have upgraded our numerical code and included a specific treatment for the halo and disk white dwarf components. In doing this the following inputs were adopted.

(1) Luminosity function: the observational white dwarf luminosity function of Liebert et al. (1988) was adopted for the disk white dwarf population whereas the theoretical luminosity functions of Isern et al. (1998) with a Salpeter-like initial mass function were adopted for the halo white dwarf component. Figure $2 b$ of this last paper shows both the disk and the halo luminosity functions for a given set of ages of the galactic halo. In the computations presented in this paper a fiducial age of the galactic halo of $12 \mathrm{Gyr}$ was adopted. 
(2) Density function: a scale height of $500 \mathrm{pc}$ (García-Berro et al. 1999) and a scale length of $2200 \mathrm{pc}$ (Chen et al. 1999) were considered for the galactic disk, while the structural parameters for Population II stars by Robin \& Crézé (1986) were adopted for the halo.

(3) Computation of $B-V$ and $V-I$ colors: the photometric calibrations of Bergeron et al. (1995) were used, adopting a mean $\log g=8.0$ for all white dwarfs and assuming that all of them have a pure hydrogen envelope (DA).

(4) Extinction vs. distance dependence: the large-scale extinction model of Hakkila et al. (1997) was adopted.

(5) Kinematical parameters: a Schwarzschild's velocity distribution was assumed with dispersions of $\left(\sigma_{\mathrm{U}}, \sigma_{\mathrm{V}}, \sigma_{\mathrm{W}}\right)=(50+0.020 z$, $35+0.015 z, 25+0.010 z) \mathrm{km} / \mathrm{s}$ for disk white dwarfs $(z$ being the distance to the galactic plane) and $(140,100,86) \mathrm{km} / \mathrm{s}$ for halo white dwarfs. The asymmetrical drift was adopted to be -25 and -220 $\mathrm{km} / \mathrm{s}$ for the galactic disk and halo, respectively. Regarding the solar motion with respect to the LSR we adopted $(10.00,5.23,7.17) \mathrm{km} / \mathrm{s}$ (Dehnen \& Binney 1998).

(6) Astrometric precision: the mean astrometric errors $\Delta \pi$ and $\Delta \mu$ were computed as a function of the Gaia magnitude $(G)$ and the ecliptic latitude following closely Lindegren (1998a). The $G$ magnitude was derived from the apparent $V$ magnitude and the observed $V-I$ color index predicted by the model, following the transformation equations given by Lindegren (1998b).

\section{GLOBAL NUMBER OF WHITE DWARFS OBSERVED BY GAIA}

Table 1 lists the number of disk and halo white dwarfs potentially observable by Gaia as predicted by our Galaxy model for the whole sky, considering limiting magnitudes of $G_{\text {lim }}=20$ and $21 \mathrm{mag}$, respectively. It is interesting to note the tremendous increase in the number of observable white dwarfs. Current catalogs of spectroscopically identified white dwarfs (McCook \& Sion 1987) have a number of objects much smaller (of the order of 1200 objects).

The number of presumably observable white dwarfs as a function of their $G$ magnitude is shown in Fig. 1 for a limiting magnitude $G_{\text {lim }}=21$. 
Table 1. Total number of white dwarfs accessible to Gaia according to our model and considering two limiting magnitudes.

\begin{tabular}{ccc}
\hline & $G_{\text {lim }}=20$ & $G_{\text {lim }}=21$ \\
\hline$N_{\text {disk }}$ & $2.3 \times 10^{5}$ & $6.7 \times 10^{5}$ \\
$N_{\text {halo }}$ & $1.1 \times 10^{3}$ & $4.2 \times 10^{3}$ \\
\hline
\end{tabular}

Figures 2 to 5 summarize our most important results when only those stars with $G \leq 20 \mathrm{mag}$ are considered. For instance, in Fig. 2 it is shown how the region of cool degenerates of the HR diagram is filled and, therefore, will be well sampled by the observations carried on by Gaia. In Fig. 3 the distance distribution of the white dwarf population accessible to Gaia is shown. It is worth noting that from this distance distribution it can be easily realized that Gaia will eventually observe disk and halo white dwarfs up to distances of about 800-1000 pc, i.e., far beyond what is actually feasible with the current technical abilities. This, when combined with robust statistical techniques (Luri et al. 1996), will undoubtedly allow a reliable determination of very important structural parameters, such as the scale height and the scale length of disk white dwarfs.

In Fig. 4 we show the absolute magnitude distribution obtained in our simulations. Also shown in Fig. 4 are the number of stars with a relative parallax error less than $1 \%$ and $10 \%$, respectively. We obtain that about 20000 disk and 100 halo white dwarfs will probably have $\Delta \pi / \pi \leq 1 \%$, with more than $70 \%$ of the stars having $\Delta \pi / \pi \leq 10 \%$ for both (disk and halo) populations. We should remark that the important fraction of stars defining the cut-off of the disk white dwarf luminosity function - those stars with luminosities of $\log \left(L / L_{\odot}\right) \simeq-4.5$ - will have a typical precision in their parallaxes better than $10 \%$.

Finally, in Fig. 5 the expected error distribution of the tangential velocities is presented. It is important to realize here that, as it is well known, a good fraction of cool white dwarfs presents a continuous spectrum without almost any relevant feature and, therefore, this distribution can be considered as the most relevant for the case under study. Considering the error distribution of Fig. 5, we can predict an unprecedented advance in our knowledge of the kinematical and dynamical evolution of the Milky Way. This possibility seems more promising when one realizes that the evolutionary time-scales of cool 


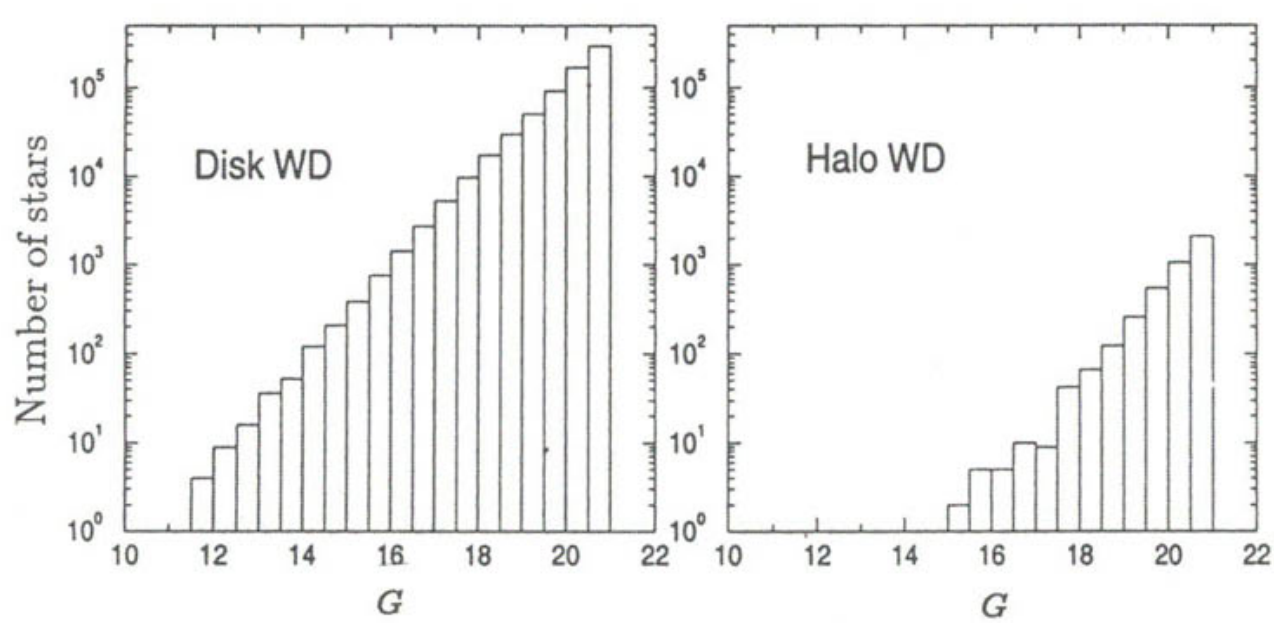

Fig. 1. Number of predicted white dwarfs in the whole sky as a function of the $G$ magnitude for $G \leq 21 \mathrm{mag}$.
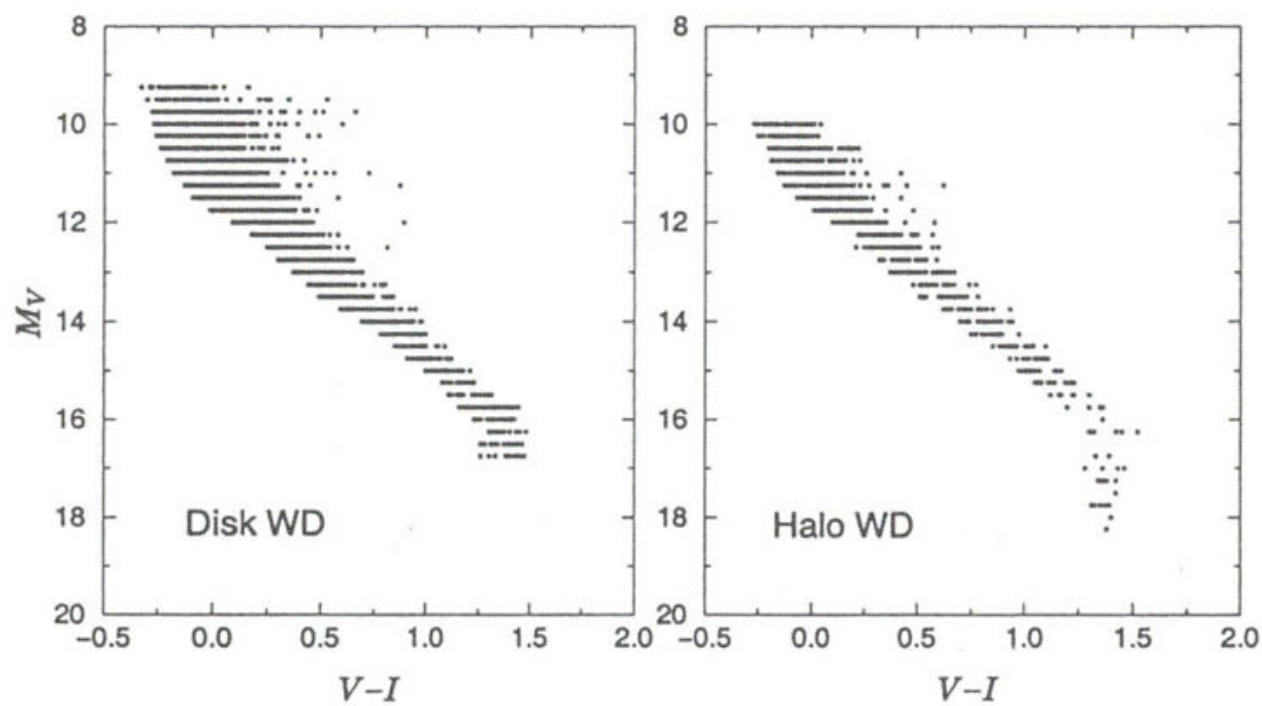

Fig. 2. HR diagram of white dwarfs with $G \leq 20 \mathrm{mag}$ (for disk white dwarfs only 5000 representative points have been plotted). 


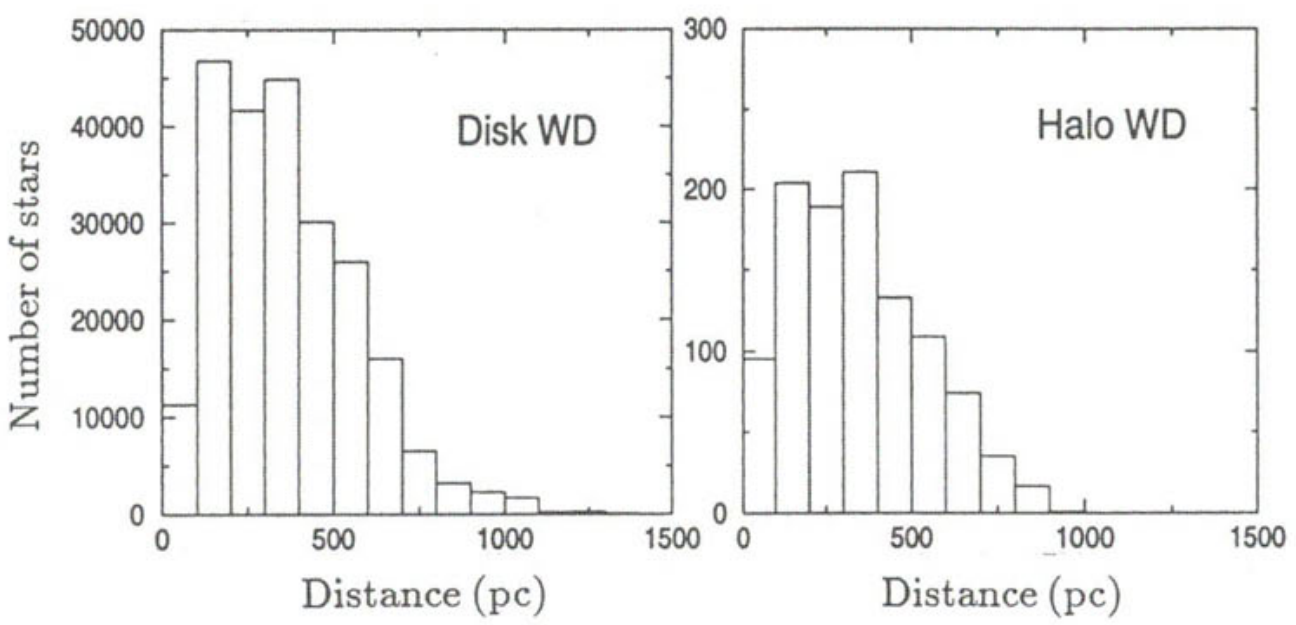

Fig. 3. Distance distribution of white dwarfs with $G \leq 20$ mag.
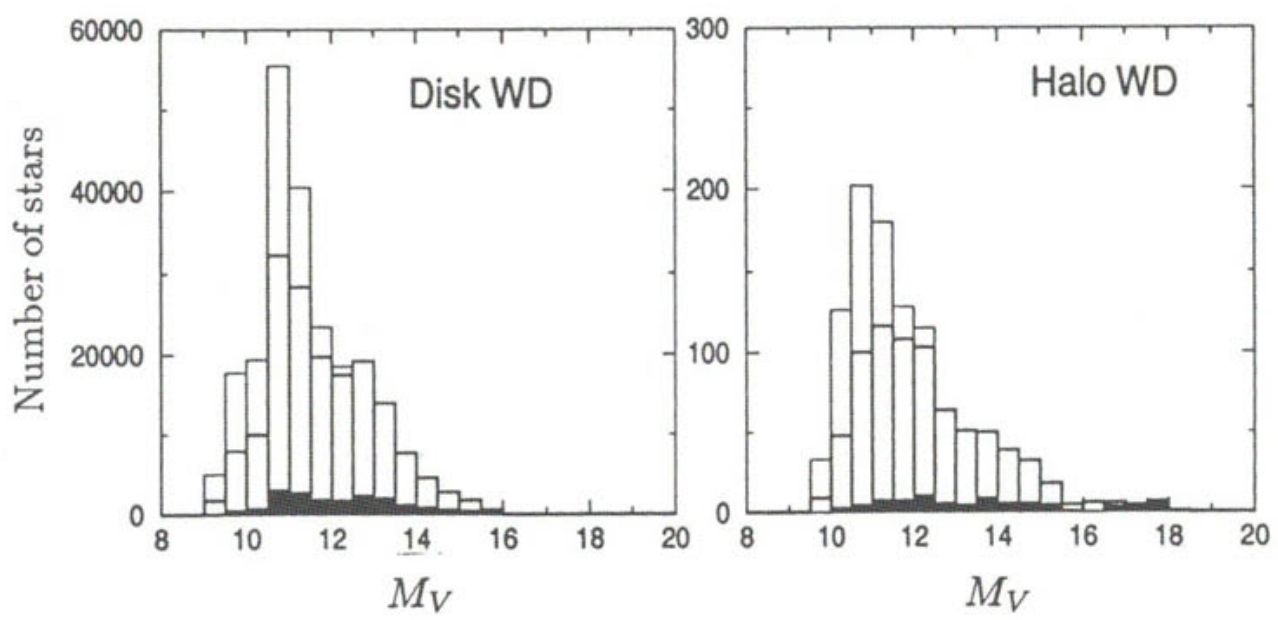

Fig. 4. Absolute magnitude distribution of white dwarfs with $G \leq$ 20 mag. The thick line corresponds to the number of stars with $\Delta \pi / \pi \leq$ $10 \%$. The filled histogram is for the number of stars with $\Delta \pi / \pi \leq 1 \%$ 

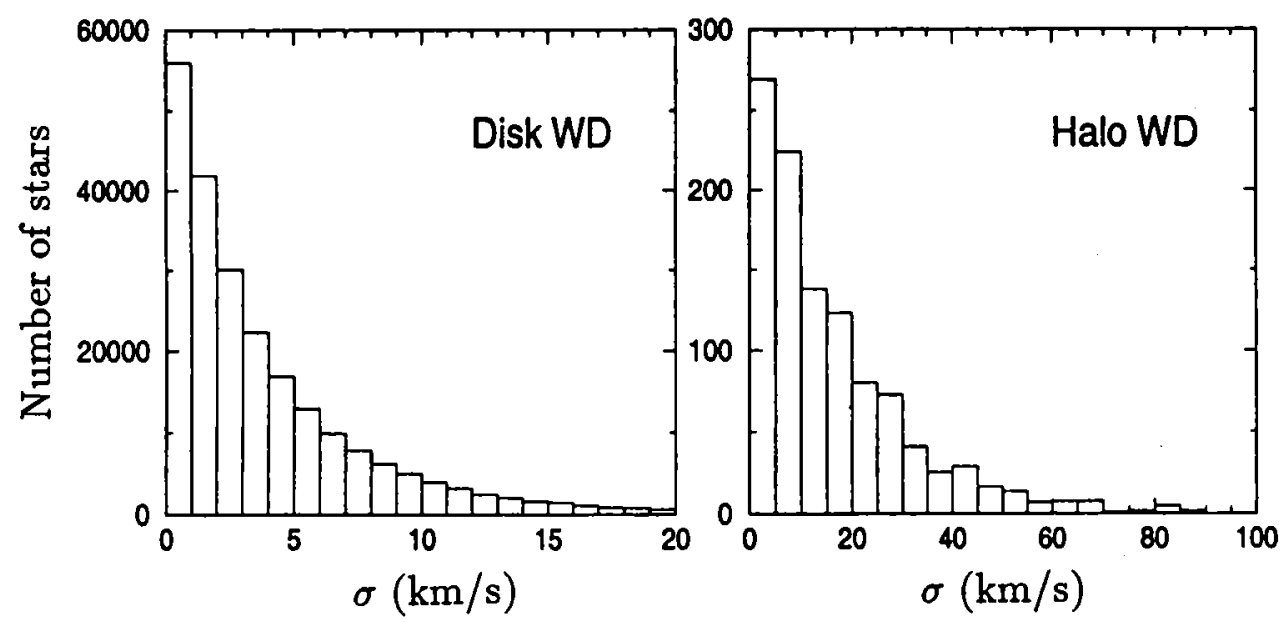

Fig. 5. Distribution of the standard error of the tangential velocity of white dwarfs with $G \leq 20$ mag.

(and therefore dim) white dwarfs are very long. This probably will allow to explore the very early epochs of star formation.

\section{SCIENCE GOALS}

The observational white dwarf database provided by Gaia will be, as shown in the previous section, orders of magnitude larger and of much better precision than those we would ever built from groundbased observations. Among other scientific goals, the following can be readily envisaged:

1. The first and most obvious scientific goal is the possibility of testing the physics of white dwarfs at extremely low temperatures. This includes both the behavior of matter under extreme pressures at very low temperatures (that is the equation of state) and the behavior of the opacities (including the role of $\mathrm{H}, \mathrm{He}$ and of metals). A check of the consistency of the currently available envelope models at very low luminosities will be also presumably attained. All this work will undoubtedly require the calculation of detailed and 
realistic cooling sequences and a careful evaluation of all the energy sources and sinks, especially during the last cooling phases, where the energetics of the envelope will be dominant.

2. One of the most successful applications of the white dwarf luminosity function has been its use as a galactic chronometer by using its observed cut-off. However, one of the major drawbacks of this technique, already pointed out by several authors (see, for instance, García-Berro et al. 1999 and references therein), is that this determination hinges on a very small number of objects and, consequently, the effects of the statistical fluctuations and of the observational procedures on the derived age of the disk can be relatively large (of the order, typically, of $1 \mathrm{Gyr}$ ). With the enormous expected increase in the observational database an optimal determination of the age of the galactic disk is also expected. Also, reliable estimates of the age and density gradients, if present, within the galactic disk could be reasonably obtained, as well as a reliable determination of the history of the SFR.

3. During the last couple of years considerable efforts have been devoted, from both the observational and the theoretical points of view, to study the nature of the recent microlensing events detected by the Macho team towards the Large Magellanic Cloud (Alcock et al. 1997). These authors suggest that perhaps up to $40 \%$ of the dark matter of the galactic halo could be in the form of white dwarfs with a mass, otherwise typical, of $0.5 M_{\odot}$. Obviously a better knowledge of the galactic halo can be envisaged through the observation of a substantial sample of nearby halo white dwarfs, which, without any kind of doubts, Gaia will achieve. This will provide us with good insights about the structure and age of our galactic halo, adding information on the objects responsible for these microlensing events, discriminating among the possible initial mass functions, and providing, for the first time, enough statistics to evaluate the intensity and duration of the bursts of star formation.

4. Other additional contributions include the following: determination of the fraction of white dwarfs in binaries, a search for the loaded gun of Type Ia SNe (WD+WD pairs which will merge within a Hubble time), and white dwarfs in open and globular clusters. 


\section{FUTURE WORK}

We plan to estimate the number of observable white dwarfs if the photometric calibrations for helium-rich envelopes are used instead of the atmospheric data for hydrogen-rich envelopes. Furthermore, we plan to introduce new photometric relations for old white dwarfs that account for the observations of white dwarfs in the Hubble Deep Field (Hansen 1998). These new calculations indicate that whereas old white dwarfs with helium dominated atmospheres are redder the cooler they are, old white dwarfs with hydrogen dominated atmospheres show a blue-loop and, therefore, could modify our predictions.

ACKNOWLEDGMENTS. This work has been supported by the CICYT under contract ESP97-1803 and by the DGES grant PB970983-C03-02.

\section{REFERENCES}

Alcock C., Allsman R. A., Alves D., Axelrod T.S., Becker A.C., Bennett D. P., Cook K.H., Freeman K. C., Griest K., Guern J., Lehner M. J., Marshall S. L., Peterson B. A., Pratt M. R., Quinn P. J., Rodgers A. W., Stubbs C.W., Sutherland W., Welch D. L. 1997, ApJ, 486, 697

Bergeron P., Wesemael F., Beauchamp A. 1995, PASP, 107, 1047

Chen B. 1997, ApJ 491, 181

Chen B., Figueras F., Torra J., Jordi C., Luri X., Galadí-Enríquez D. 1999, A\&A, submitted

Dehnen W., Binney J. J. 1998, MNRAS, 298, 387

Díaz-Pinto A., García-Berro E., Hernanz M., Isern J., Mochkovitch R. 1994, A\&A, 282, 86

García-Berro E., Hernanz M., Mochkovitch R., Isern J., 1988, A\&A, 193, 141

García-Berro E., Torres S., Isern J., Burkert A. 1999, MNRAS, 302, 173 Hakkila J., Myers J.M., Stidham B. J., Hartmann D. H., 1997, AJ, 114, 2043

Hansen B. M. S. 1998, Nature, 394, 860

Hernanz M., García-Berro E., Isern J., Mochkovitch R., Segretain L., Chabrier G. 1994, ApJ, 434, 652

Isern J., García-Berro E., Hernanz M., Mochkovitch R., Burkert A. 1995a, in White Dwarfs, eds. D. Koester \& K. Werner, Springer Verlag, p. 19 
Isern J., García-Berro E., Hernanz M., Mochkovitch R., Burkert A. 1995b, in The Formation of the Milky Way, eds. E. J. Alfaro \& A. J. Delgado, Cambridge University Press, p. 19

Isern J., García-Berro E., Hernanz M., Mochkovitch R., Torres S. 1998, ApJ, 503, 239

Liebert J., Dahn C. C., Monet D. G. 1988, ApJ, 332, 891

Lindegren L. 1998a, SAG-LL-023 (private communication)

Lindegren L., 1998b, SAG-LL-017 (private communication)

Lindegren L., Perryman M. A.C. 1997, in Proc. ESA Symp. Hipparcos Venice '97, eds. B. Battrick, M. A. C. Perryman \& P. L. Bernacca, ESA SP-402, p. 799

Luri X., Mennessier M.O., Torra J., Figueras F. 1996, A\&A, 117, 405

McCook G. P., Sion E. M. 1987, ApJS, 65, 603

Noh H.-R., Scalo J. 1990, ApJ, 352, 605

Robin A., Crézé M. 1986, A\&A, 157, 71

Torra J., Chen B., Figueras F., Jordi C., Luri X. 1999, Baltic Astronomy, 8,171 (this volume)

Winget D.E., Hansen C.J., Liebert J., Van Horn H.M., Fontaine G., Nather R., Kepler S. O., Lamb D. K. 1987, ApJ, 315, L77 